TITLE:

\title{
Impairment of unconscious, but not conscious, gaze-triggered attention orienting in Asperger's disorder
}

\section{$\operatorname{AUTHOR}(\mathrm{S})$ :}

Sato, Wataru; Uono, Shota; Okada, Takashi; Toichi, Motomi

\section{CITATION:}

Sato, Wataru ...[et al]. Impairment of unconscious, but not conscious, gaze-triggered attention orienting in Asperger's disorder. Research in Autism Spectrum Disorders 2010, 4(4): $782-786$

ISSUE DATE:

2010-10

URL:

http://hdl.handle.net/2433/130702

\section{RIGHT:}

(C) 2010 Elsevier Ltd; この論文は著者最終稿です。内容が印刷版と異な ることがありますので、引用の際には出版社版をご確認ご利用くださ い。 This is the Accepted Author Manuscript. Please cite only the published version. 


\section{Impairment of unconscious, but not conscious, gaze-triggered attention orienting in Asperger's disorder}

Wataru Sato ${ }^{1}$, Shota Uono ${ }^{2}$, Takashi Okada ${ }^{3}$, and Motomi Toichi ${ }^{4}$

1 Department of Comparative Study of Cognitive Development (funded by

Benesse Corporation), Primate Research Institute, Kyoto University, Inuyama, Aichi 484-8506, Japan.

2 Department of Cognitive Psychology in Education, Graduate School of Education, Kyoto University, Yoshida-honmachi, Sakyo-ku, Kyoto, 606-8501, Japan

3 Department of Neuropsychiatry, Faculty of Medicine, Kyoto University, 54 Shogoin-Kawaharacho, Sakyo-ku, Kyoto 606-8507, Japan.

4 Faculty of Human Health Science, Graduate School of Medicine, Kyoto University, 53 Shogoin-Kawaharacho, Sakyo-ku, Kyoto 606-8507, Japan.

\section{Corresponding author}

Wataru Sato

Department of Comparative Study of Cognitive Development (funded by Benesse Corporation), Primate Research Institute, Kyoto University, Inuyama, Aichi 484-8506, Japan.

E-mail: sato@pri.kyoto-u.ac.jp 


\section{Abstract}

Impairment of joint attention represents the core clinical features of pervasive developmental disorders (PDD), including autism and Asperger's disorder. However, experimental studies reported intact gaze-triggered attentional orienting in PDD. Since all previous studies employed supraliminal presentation of gaze stimuli, we hypothesized that individuals with PDD may be impaired not in conscious but in unconscious gaze-triggered attention shift. We tested the hypothesis in a group of Asperger's disorder $(\mathrm{N}=12)$ and age- and gender-matched controls $(\mathrm{N}=13)$, using a cueing paradigm with supraliminal and subliminal presentation of gaze cues. Under supraliminal conditions, the gaze cueing effect was evident in both groups. Under subliminal conditions, the Asperger group, unlike the control group, did not show the gaze cueing effect. These results indicate the impairment of unconscious, but not conscious, joint attention in Asperger's disorder, which may underlie some clinical findings of social malfunction in PDD.

Keywords: Attention orienting; Asperger's disorder; Gaze; Pervasive developmental disorder (PDD); Unconscious processing; Subliminal presentation. 


\section{Introduction}

Individuals with pervasive developmental disorders (PDD), including autism and Asperger's disorder, are characterized primarily by qualitative impairments of social interaction (American Psychiatric Association, 2000; Matson, Compton, \& Sevin, 1991). One of the most evident features of their social impairment is the deficit in joint attention (Mundy, Sigman, \& Kasari, 1994). For example, when the attending physician suddenly averts his gaze to look at environmental objects during a clinical interview, an individual with PDD fails to follow his gaze direction (Okada, Sato, Murai, Kubota, \& Toichi, 2003).

In contrast to such obvious clinical evidence of impaired joint attention, several experimental studies have found a normal ability to shift attention with another's gaze reflexively in PDD (Chawarska, Klin, \& Volkmar, 2003; Johnson et al., 2005; Kylliainen \& Hietanen, 2004; Okada et al., 2003; Senju, Tojo, Dairoku, \& Hasegawa, 2004;

Swettenham, Condie, Campbell, Milne, \& Coleman, 2003; Vlamings, Stauder, van Son, \& Mottron, 2005; for a review see Nation \& Penny, 2008). The studies have used Posner's (1980) cueing paradigm to examine joint attention (c.f., Frischen, Bayliss, \& Tipper, 2007). For example, Okada et al. (2003) presented a face with eyes directed left or right to individuals with $\mathrm{PDD}$, and to controls with no developmental disorder. Then, a target appeared to the right or left side of the face. The reaction time ( $\mathrm{RT}$ ) to detect the target was shorter at a validly cued location than at an invalidly cued location in both PDD and control participants. These results suggest that computerized experiments using 
a conventional gaze cueing paradigm cannot reveal the impaired joint attention in PDD.

Experimental social psychological studies have revealed that our social interactions are full of adaptive unconscious processes (Wilson, 2002). A recent study revealed that gaze-triggered attention could even occur unconsciously (Sato, Okada, \& Toichi, 2007). Based on these data, we hypothesized it would be unconscious, rather than conscious, gaze-triggered attention shift that is impaired in PDD. Here we tested this hypothesis in a group of Asperger's disorder and age- and gender-matched typically developing controls. We used the same cueing paradigm with supraliminally or subliminally presented gaze cues, as in a previous study (Sato et al., 2007).

\section{Methods}

\subsection{Participants}

The Asperger group ( 3 females, 9 males; mean $\pm S D$ age $=17.2 \pm 6.3$ years) consisted of 11 (2 females, 9 males) with Asperger's disorder and 1(female) with PDD not otherwise specified (PDD-NOS), who did not satisfy all the diagnostic criteria for Asperger's disorder but exhibited mild symptoms of PDD. The diagnoses, based on the DSM-IV-TR (American Psychiatric Association, 2000), were made by psychiatrists with expertise in developmental disorders. Neurological and psychiatric problems other than those associated with PDD were ruled out. Participants were taking no medication. The full-scale IQ, measured by the WAIS-R or WISC-R, of all participants in the Asperger group scored in the normal range (Full-scale 
IQ $=106.8 \pm 9.3 ;$ Verbal IQ $106.4 \pm 13.1$; Performance IQ 104.2 \pm 10.0 )

Participants in the control group ( 3 females, 10 males; mean $\pm S D$ age $=$

$19.7 \pm 1.9$ ) were matched for age and gender with the Asperger group. All participants had normal or corrected-to-normal visual acuity. After the procedure and purpose of the study were explained fully and before testing, written informed consent was obtained from the participants or their parents.

\subsection{Experimental design}

The experiment was constructed as a two-factorial mixed randomized-repeated design, with group (Asperger or control) as the randomized factor, and presentation condition (subliminal or supraliminal) as the repeated factor.

\subsection{Apparatus}

The events were controlled by SuperLab Pro 2.0 (Cedrus) and implemented on a Windows computer (MA55J, NEC). The stimuli were presented on a 19-inch CRT monitor (GDM-F400, Sony) with a refresh rate of $100 \mathrm{~Hz}$ and a resolution of $1024 \times 768$ pixels. The participants' responses were recorded using a response box (RB-400, Cedrus).

\subsection{Stimuli}

The gaze cues consisted of schematic faces in which the eye gaze was directed toward either the left or right. Masks were mosaic patterns that covered all of the facial features of the cue stimuli. The cues and masks subtended $6.5^{\circ}$ vertically $\times 6.5^{\circ}$ horizontally. The target was an open circle subtending $1.0^{\circ}$ vertically $\times 1.0^{\circ}$ horizontally. These stimuli consisted of a 
black line drawing on a white background.

\subsection{Procedure}

The procedure was identical to that of a previous study (Sato et al., 2007). The experiments were conducted individually in a small room. The participant was seated comfortably with her/his head supported by a chin-and-forehead rest located $0.57 \mathrm{~m}$ from the screen.

A threshold assessment session was first conducted. The stimulus onset asynchrony (SOA) between the target and mask was manipulated. To assess the upper limit of SOA for subliminal presentation in each participant, blocks of 20 subliminal cue presentation trials, i.e., 10 each for the left and right gaze directions, were prepared. In each trial, after the presentation of a fixation point, i.e., a small black "+" lasting $680 \mathrm{~ms}$, the gaze cue was presented in the center of the monitor, after which the mask was presented in the same location. The presentation time of the mask was adjusted so that the total presentation period of the gaze cue and the mask was $200 \mathrm{~ms}$. The order of gaze direction was randomized. The participant was asked to orally answer the question, "Did you see the gaze? If so, report the direction of the gaze." They were also asked not to guess at answers. The participants responded either "Yes" or "No," and in the case of the former, they then reported the gaze direction that they had seen. Starting with $10 \mathrm{~ms}$, the SOA was prolonged by $10 \mathrm{~ms}$ increments. After the participants finished each block, the performance was investigated. If the participant correctly recognized at least 1 of the 20 stimuli, the corresponding SOA was regarded as the lower limit of conscious awareness for the cue for that participant, and an SOA $10 \mathrm{~ms}$ shorter than that limit was 
used in the trial session. The mean $( \pm S D)$ SOA was as $19.2 \pm 10.9$ and 14.7 $\pm 7.8 \mathrm{~ms}$ for the Asperger and control groups, respectively (two-tailed $t$-test, $t(23)=1.21, n . s)$.

The trial session was then conducted. The participant completed a total of 144 trials, presented in two blocks of 72 . Each block contained an equal number of valid and invalid trials for each presentation condition. The order of cue validity was randomized within each block. The order of presentation condition was counterbalanced across participants. At the beginning of each block, the participant received 10 practice trials. A short break was interposed after 36 trials in each block, and a longer break was interposed after each block.

For each trial (Fig. 1), a fixation point, i.e., a small black “+," was presented for $680 \mathrm{~ms}$ at the center of the screen. The gaze cue was then presented at the same location. Subsequently, a target was presented in either the left or right visual field $\left(5.0^{\circ}\right.$ apart from the center $)$ until a response was made. The participant was instructed to specify as quickly as possible whether the target appeared on the left or right side of the screen by pressing the corresponding key on the switch box using the left or right index finger, respectively.

$$
* * * * * * * * * * * * * * * * * * * * * *
$$

Fig. 1

$* * * * * * * * * * * * * * * * * * * * * *$

After the completion of all trials, debriefing was conducted and the participant was asked whether she/he had consciously perceived the gaze cues in the subliminal presentations. We confirmed that none of the participants had consciously perceived the gaze cues in the subliminal 
presentations.

\subsection{Data analysis}

The median correct reaction time (RT) under each condition was calculated for each participant. The differences in RT between valid and invalid conditions were then calculated as a measure of the gaze cueing effect as in previous studies (e.g., Okada, Sato, \& Toichi, 2006). The RT differences were analyzed using a 2 (group: Asperger or control) $\times 2$ (presentation condition: subliminal or supraliminal) analysis of variance (ANOVA). For significant interactions, follow-up multiple comparisons were conducted for the group factor using $t$-tests (two-tailed) with the Bonferroni correction. One-sample $t$-tests (two-tailed) were also performed to test for differences from zero with the Bonferroni correction.

Preliminary analyses were conducted for error percentages. The error rates were small $(<5 \%)$ and there was no evidence of a speed-accuracy trade-off phenomenon. Hence, we report only the RT results.

\section{Results}

The ANOVA for the differences in RT between validly and invalidly cued conditions (Fig. 2) revealed a significant interaction of group $\times$ presentation condition $(F(1,23)=5.90, p<.05)$. The main effect of presentation condition was also significant $(F(1,23)=38.88, p<.001)$.

$$
\begin{gathered}
* * * * * * * * * * * * * * * * * * * * * * \\
\text { Fig. } 2 \\
* * * * * * * * * * * * * * * * * * * * * *
\end{gathered}
$$

Follow-up analyses for the interaction revealed that there was a 
significant between-group difference in the subliminal condition $(t(23)=$ 3.33, $p<.001$ ), which indicated a larger RT difference for the control group than for the Asperger group. There was no significant between-group difference in the supraliminal condition $(t(23)=1.34$, n.s. $)$.

Bonferroni-corrected one-sample $t$-tests were performed to test for differences from zero. All conditions differed significantly from zero $(\underline{t} \mathrm{~s}>$ $2.87, p$ s <.05), with the exception of subliminal presentations to the Asperger group $(t(11)=0.92, n . s$.$) .$

\section{Discussion}

Congruent with previous studies that used the supraliminal presentation of gaze cues (Nation \& Penny, 2008), we found a gaze cueing effect for both the Asperger and control groups under supraliminal conditions. These data confirm that conscious gaze-triggered attention orienting is not impaired in individuals diagnosed with PDD.

Under subliminal conditions, however, there was a gaze cueing effect in the control group, but not in the Asperger group. The triggering of attention orientation in participants without developmental disorders by the unconscious gaze cue is consistent with previous results (Sato et al., 2007). The impairment in the orienting response triggered by an unconscious gaze cue in Asperger's disorder is a novel finding. This finding seems consistent with previous behavioral studies that have reported impairment in the unconscious processing of facial stimuli in individuals with PDD (e.g., Hall, West, \& Szatmari, 2007). The results support the hypothesis that individuals with PDD have impaired 
unconscious, but not conscious, gaze-triggered attention.

Our results can explain the discrepancy between previous clinical (Mundy et al., 1994) and experimental (Nation \& Penny, 2008) findings on joint attention in PDD. Psychophysical studies have shown that, contrary to what intuition might suggest, humans consciously perceive only very restricted areas within the range of areas available for immediate attention (Simons \& Rensink, 2005). Consistent with this notion, psychological studies have indicated that social behaviors are heavily influenced by unconscious processing (Wilson, 2002). In particular, previous research has found that gaze-triggered attention orienting occurs unconsciously (Sato et al., 2007). Thus, individuals that exhibit typical developmental milestones have at least two mechanisms to achieve automatic joint attention: conscious processing of the gazes of others that occur within restricted attended areas and unconscious processing of the gazes of others that occur within broader unattended areas. Our results indicate that individuals with PDD have access to only a single conscious mechanism for the achievement of joint attention; therefore, these individuals may fail to show joint attention in relation to individuals outside of the range of conscious attention.

Our finding of impaired unconscious gaze processing in individuals diagnosed with PDD corroborates evidence from neuroscientific literature. A neuroimaging study of typically developing participants reported the involvement of the amygdala in the unconscious processing of gaze (Whalen et al., 2004). A study of patients with unilateral amygdala incisions indicated that the amygdala is involved in gaze-triggered 
attention orienting (Okada et al., 2008). Considering the neural network from which the amygdala receives visual input, i.e., the subcortical pathway via the pulvinar and superior colliculus (Adolphs, 2002), it is possible that the amygdala processes the information derived from gaze, even before conscious awareness has emerged. Postmortem histopathological (e.g., Schumann \& Amaral, 2006) and neuroanatomical imaging (e.g., Schumann et al., 2004) studies have reported a pronounced abnormality of the amygdala in individuals diagnosed with PDD. Neuroimaging studies have reported that these individuals show reduced activity of the amygdala in the processing of gaze (e.g., Baron-Cohen et al., 1999). These data suggest that dysfunction of the amygdala may be the neural background of the impairment of the unconscious gaze-triggered attention orienting in individuals with PDD.

In contrast, the conscious awareness of visual stimuli is implemented in the cortical visual areas (Treisman \& Kanwisher, 1998). Neuroimaging studies in normatively developing participants showed the activation of some cortical visual areas, including the superior temporal sulcus (STS) region, in response to supraliminally presented gaze (e.g., Hoffman \& Haxby, 2000). A neuroimaging study in individuals with PDD also reported the activation of the STS region in the conscious processing of gaze (Baron-Cohen et al., 1999). These data suggest that the cortical pathways involved in the conscious processing of gaze are not impaired in PDD.

Controversy persists about whether automatic processing can be identified with the absence of consciousness (Tzelgov, 1997). Our results indicate that automatic gaze-triggered attention consists of conscious and 
Res Autism Spectr Disord 12

unconscious processes, with one dissociable from the other. It has been proposed that automatic processes could derive from either heredity or practice (Hasher \& Zacks, 1979). We speculate that individuals with PDD may have innate impairments in the unconscious subcortical system, but can acquire, through practice, the conscious cortical system that allows joint attention.

In summary, our results showed gaze-triggered attentional orienting for both the Asperger and control groups under supraliminal conditions; however, the Asperger group, unlike the control group, did not show the gaze cueing effect under subliminal conditions. These results indicate the impairment of unconscious, but not conscious, joint attention in Asperger's disorder, which may underlie some clinical findings of social malfunction in PDD. 
Res Autism Spectr Disord 13

\section{References}

Adolphs, R. (2002). Neural systems for recognizing emotion. Current opinion in neurobiology, 12, 169-177.

American Psychiatric Association (2000). Diagnostic and statistical manual for mental disorders (DSM-IV-TR). Washington, DC: APA.

Baron-Cohen, S., Ring, H. A., Wheelwright, S., Bullmore, E. T., Brammer, M. J., Simmons, A., et al. (1999). Social intelligence in the normal and autistic brain: An fMRI study. European Journal of Neuroscience, 11, 1891-1898.

Chawarska, K., Klin, A., \& Volkmar, F. (2003). Automatic attention cueing through eye movement in 2 -year-old children with autism. Child Development, 74, 1108-1122.

Frischen, A. Bayliss, A. P. Tipper, S. P. (2007). Gaze cueing of attention: visual attention, social cognition, and individual differences. Psychological Bulletin, 133, 694-724.

Hall, G. B., West, C. D., \& Szatmari, P. (2007). Backward masking: Evidence of reduced subcortical amygdala engagement in autism. Brain and cognition, 65, 100-106.

Hasher, L., \& Zacks, R. T. (1979). Automatic and effortful processes in memory. Journal of experimental psychology. General, 108, $356-388$.

Hoffman, E. A., \& Haxby, J. V. (2000). Distinct representations of eye gaze and identity in the distributed human neural system for face perception. Nature Neuroscience, 3, 80-84. 
Johnson, M. H., Griffin, R., Csibra, G., Halit, H., Farroni, T., De Haan, M., et al. (2005). The emergence of the social brain network: Evidence from typical and atypical development. Development and Psychopathology, 17, 599-619.

Kylliainen, A., \& Hietanen, J. K. (2004). Attention orienting by another's gaze direction in children with autism. Journal of Child Psychology and Psychiatry, 45, 435-444.

Matson, J. L., Compton, L. S., \& Sevin, J. A. (1991). Comparison and item analysis of the MESSY for autistic and normal children. Research in Developmental Disabilities, 12, 361-369.

Mundy P, Sigman M, Kasari C (1994). The theory of mind and joint-attention deficits in autism. In $\mathrm{S}$. Baron-Cohen \& $\mathrm{H}$. Tager-Flusberg (eds.), Understanding Other Minds: Perspectives from Autism (pp. 181-203). New York: Oxford University Press.

Nation, K., \& Penny, S. (2008). Sensitivity to eye gaze in autism: is it normal? Is it automatic? Is it social? Development and Psychopathology, 20, 79-97.

Okada, T., Sato, W., Murai, T., Kubota, Y. \& Toichi, M. (2003). Eye gaze triggers visuospatial attentional shift in individuals with autism. Psychologia, 46, 246-254.

Okada, T., Sato, W., \& Toichi, M. (2006). Right hemispheric dominance in gaze-triggered reflexive shift of attention in humans. Brain and Cognition, 62, 128-133.

Okada T., Sato, W., Kubota, Y., Usui, K., Inoue, Y., Murai, T., et al. (2008). Involvement of medial temporal structures in reflexive 
Res Autism Spectr Disord 15

attentional shift by gaze. Social Cognitive and Affective Neuroscience, 3, 80-88.

Posner, M.I. (1980). Orienting of attention. Quarterly Journal of Experimental Psychology, 32, 3-25.

Sato, W., Okada, T., \& Toichi, M. (2007). Attentional shift is triggered by gaze without awareness. Experimental Brain Research, 183, 87-94.

Schumann, C. M., \& Amaral, D. G. (2006). Stereological analysis of amygdala neuron number in autism. Journal of Neuroscience, 26, $7674-7679$.

Schumann, C. M., Hamstra, J., Goodlin-Jones, B. L., Lotspeich, L. J., Kwon, H., Buonocore, M. H., et al. (2004). The amygdala is enlarged in children but not adolescents with autism; Hippocampus is enlarged at all ages. Journal of Neuroscience, 24, $6392-6401$.

Senju, A., Tojo, Y., Dairoku, H., \& Hasegawa, T. (2004). Reflexive orienting in response to eye gaze and an arrow in children with and without autism. Journal of Child Psychology and Psychiatry, $45,445-458$.

Simons, D. J., \& Rensink, R. A. (2005). Change blindness: Past, present, and future. Trends in Cognitive Sciences, 9, 16-20.

Swettenham, J., Condie, S., Campbell, R., Milne, E., \& Coleman, M. (2003). Does the perception of moving eyes trigger reflexive visual orienting in autism? Philosophical Transactions of the Royal Society of London Series B: Biological Sciences, 358, 
Res Autism Spectr Disord 16

$325-334$.

Tzelgov, J. (1997). Specifying the relations between automaticity and consciousness: A theoretical note. Consciousness and Cognition, $6,441-451$.

Treisman, A. M., \& Kanwisher, N. G. (1998). Perceiving visually presented objects: recognition, awareness, and modularity. Current opinion in neurobiology, 8, 218-222.

Vlamings, P., Stauder, J. E. A., van Son, I. A. M., \& Mottron, L. (2005). Atypical visual orienting to gaze- and arrow-cues in adults with high functioning autism. Journal of Autism and Developmental Disorders, 35, 267-277.

Whalen, P. J., Kagan, J., Cook, R. G., Davis, F. C., Kim, H., Polis, S., McLaren, D. G., Somerville, L. H., McLean, A. A., Maxwell, J. S., \& Johnstone, T. (2004). Human amygdala responsivity to masked fearful eye whites. Science, 306, 2061.

Wilson, T. D. (2002). Strangers to ourselves: Discovering the adaptive unconscious. Cambridge: Belknap Press of Harvard University Press. 
Supraliminal

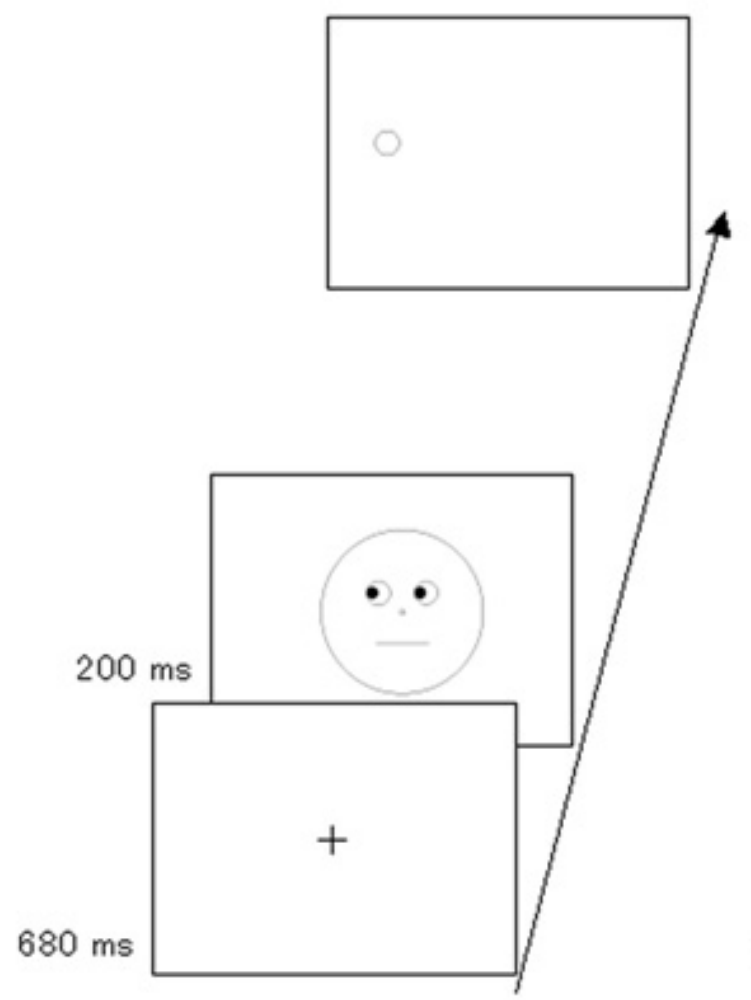

Subliminal

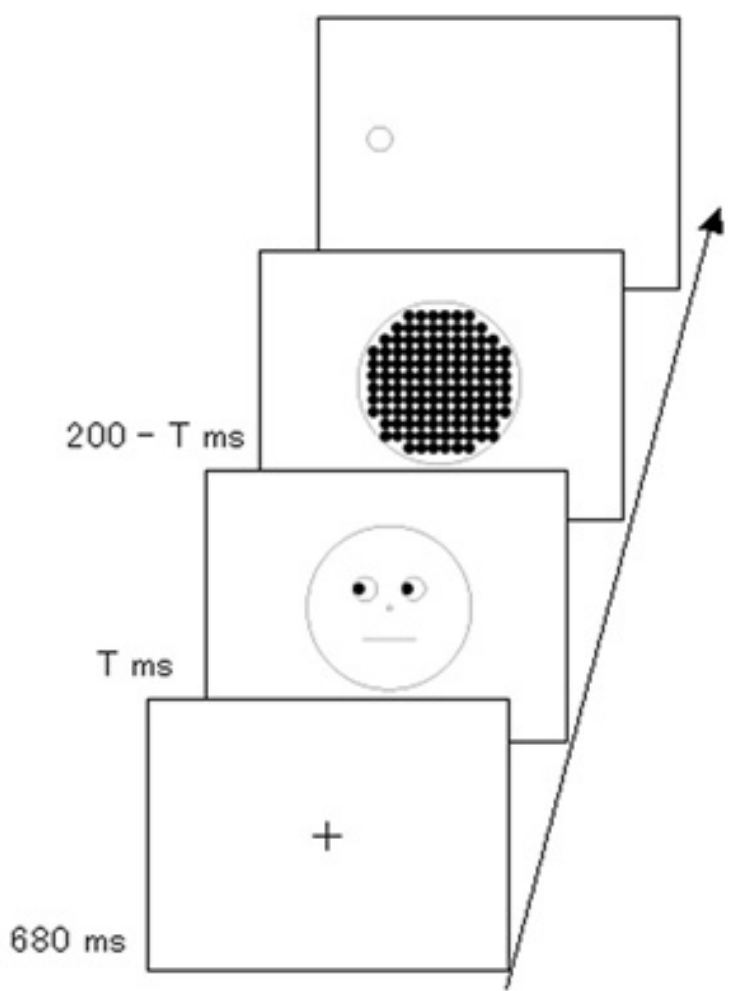

Fig. 1. Illustrations of stimulus presentations. In the subliminal presentation, the presentation time of the gaze cue (T) was adjusted for each participant's threshold and the presentation period of the mask was also adjusted so that the total period of the gaze cue and the mask was 200 ms. 
Res Autism Spectr Disord 18

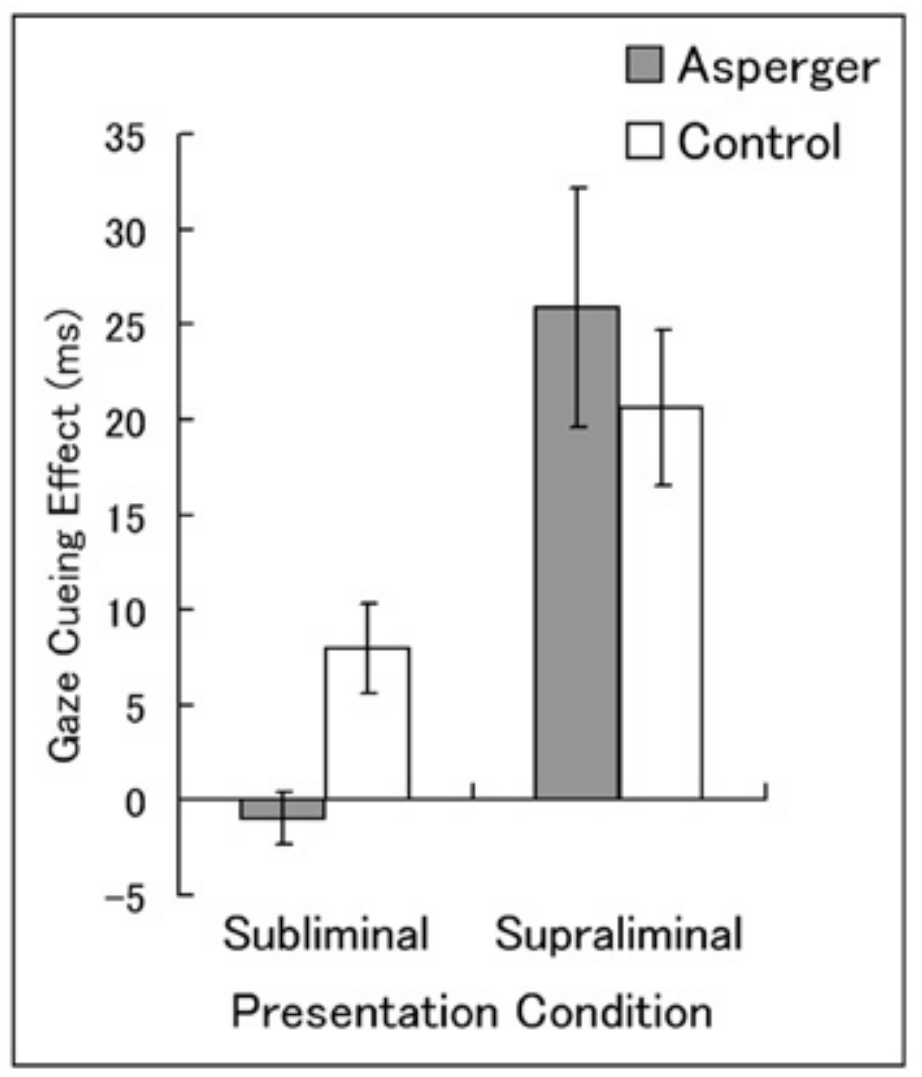

Fig. 2. Mean (with SE) gaze cueing effect (i.e., differences in reaction time between validly and invalidly cued conditions). 